\title{
The MindLab Project. Local Museums Supporting Community Wellbeing Before and After UK Lockdown
}

\author{
Jade French, Nic Lunt, Martin Pearson
}

\begin{abstract}
Catalyst is a science discovery centre and independent museum based in Widnes, United Kingdom. Since 2018, it has collaborated with mental health charity Mind Halton on MindLab, a science-inspired wellbeing project for local residents in an area with low access to mental health support. As twenty-first century museums are being increasingly regarded as community resources capable of producing valuable social impact, this article considers the role of museums in harnessing this potential to enhance community health and wellbeing during the COVID-19 pandemic. It reflects on the challenges encountered during this unprecedented lockdown, as well as the emerging opportunities, through the perspectives of those delivering MindLab.
\end{abstract}

\section{Introduction}

The coronavirus (COVID-19) pandemic is having a profound effect on all aspects of society. Already, it is evident that direct and indirect psychological and social effects are widespread, with data revealing that over half (53.1 per cent) of British people have reported that it is affecting their wellbeing. ${ }^{1}$ With increased feelings of stress, anxiety and isolation, many are seeking additional support in their local communities.

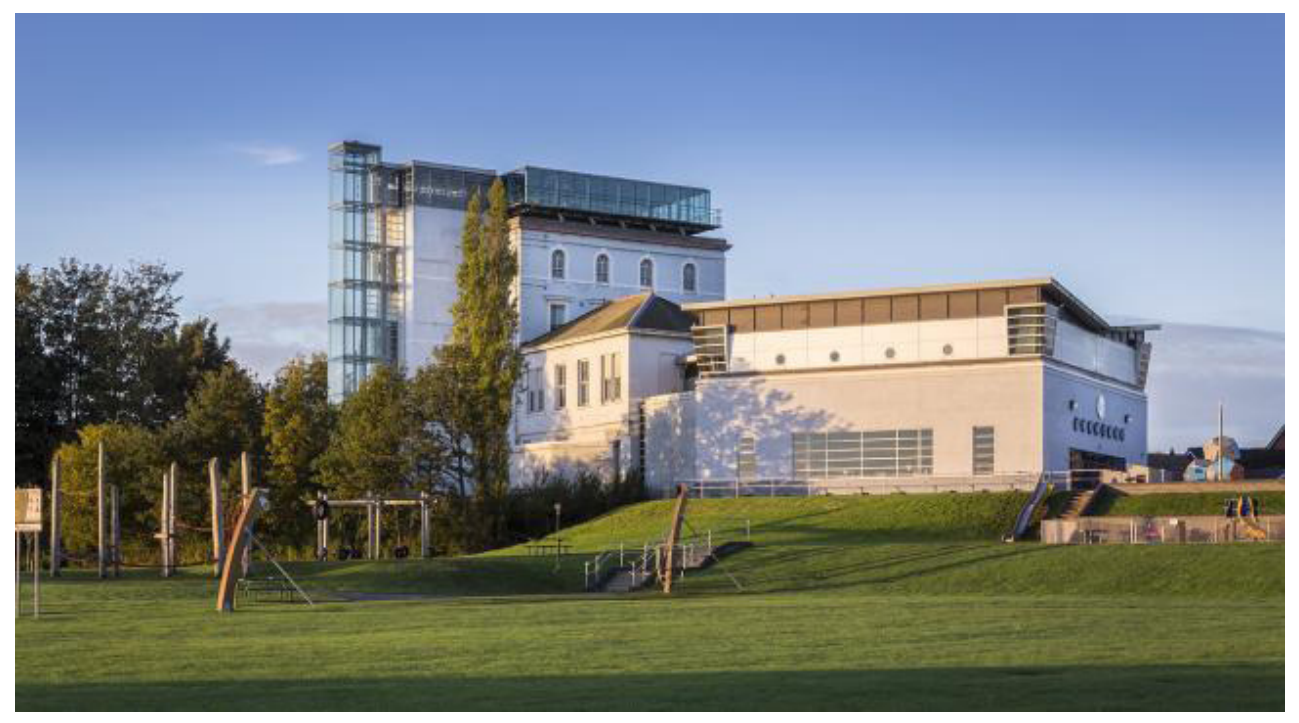

Image 1. Catalyst Science Discovery Centre 
Founded in 1989, Catalyst is a small, local science discovery centre and independent museum which explores the science and technology of the chemical industry and its impact on society past and present. The primary audiences for Catalyst are children and families, reflected in its thirty-year track record of working with schools, an established public education programme and hands-on science exhibits. However, since its 2018 capital redevelopment, ${ }^{2}$ Catalyst has introduced its first community engagement programme, which seeks to expand its current audience. Mental health and wellbeing were identified as significant issues in the local community and became a focus for the museum's expanded mission.

Significantly, Catalyst is located in the industrial town of Widnes, Cheshire, where its neighbourhood is among the 10 per cent most economically deprived areas nationally. ${ }^{3}$ This supports a body of research indicating there is a social gradient to wellbeing whereby individuals from poorer socio-economic backgrounds experience more challenges to their mental health. ${ }^{4}$ This leaves local counselling services overwhelmed, with many people unable to access other forms of support. In this context, Catalyst partnered with local mental health charity Mind Halton to explore alternative ways to support people living with a mental health condition, launching MindLab in 2018. Mindlab offers participants a dual frame for mental health issues and wellbeing - a therapeutic one and a scientific one.

In its first year, the project proved successful, with excellent uptake and participant feedback. Due to the pandemic it was paused indefinitely in March 2020, leaving the team grappling with how to continue and adapt this project at a time when it is needed most. This article reflects on the challenges encountered during the lockdown, as well as the emerging opportunities, through the perspectives of those delivering MindLab services. By sharing

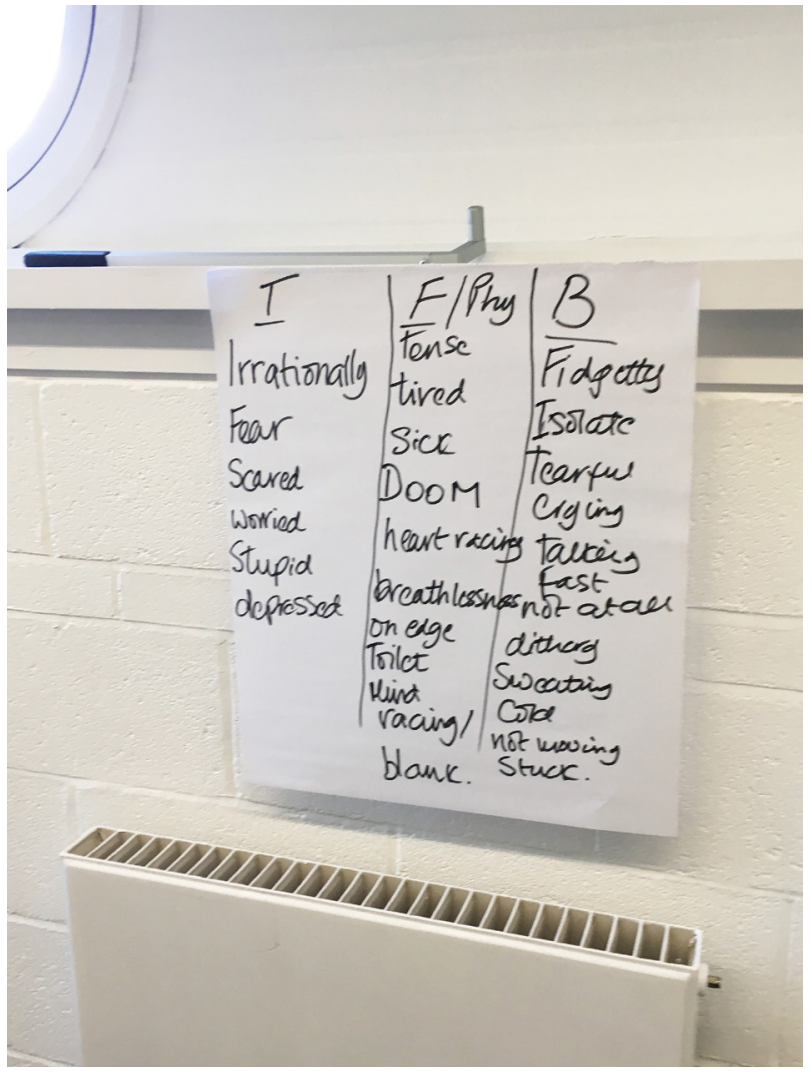

Image 2. Cognitive behavioural therapy session reviewing 'thoughts', 'feelings' and 'behaviours' these experiences, we hope to demonstrate how museums and science centres can play a role in supporting community wellbeing during the pandemic, as well as contributing to studying the importance of mental health interventions in museology more broadly.

\section{Before: The MindLab Project}

The project was designed by Nic Lunt, Chief Officer of Mind Halton, in collaboration with Jade French, Catalyst's Community Engagement Coordinator with support from Lucinda Lewis, Catalyst's Education Manager. Capitalizing on the specialist resources within Catalyst, including a lab, classrooms, interactive museum exhibitions, a planetarium and an observatory, the project aims to incorporate science discovery into Mind Halton'smental health programme for people in the local community. It utilizes Cognitive Behavioural Therapies (CBT) to assist local citizens with current problems. The first four-week programme was launched during August 2019 on the theme of 'anxiety', 
followed by a second five-week programme on 'resilience' in January 2020. Participants were recruited via Mind Halton and comprised existing users and others who have been referred by the local authority.

In a typical session before the pandemic, a counsellor from Mind Halton would begin by framing a theme such as 'emotional pressures'. The counsellor led the group through an hour-long discussion using CBT techniques like STOPP, an acronym for 'stop and step back', 'take a breath', 'observe', 'pull back: put in some perspective' and 'practice what works: proceed'. The workshop was then handed over to Catalyst's Education team, who invited participants to think about the theme differently - through science discovery. During the 'emotional pressures' workshop, for example, the group investigated the concept of pressure through experiments with chemical reactions, vacuums and syringes in Catalyst's lab, drawing parallels with insights gained through earlier discussion.

Integrating science discovery into CBT presents pathways to practise the 'Five Ways to Wellbeing's (Connect, Be Active, Take Notice, Keep Learning, Give). Participants connect with others during experiments, facilitating social experiences that reduce isolation. Additionally, activities encourage participants to notice their surroundings, such as the lab, and provide learning opportunities that increase self-esteem. Participants also describe how the activities and museum setting provide a positive distraction from clinical environments.

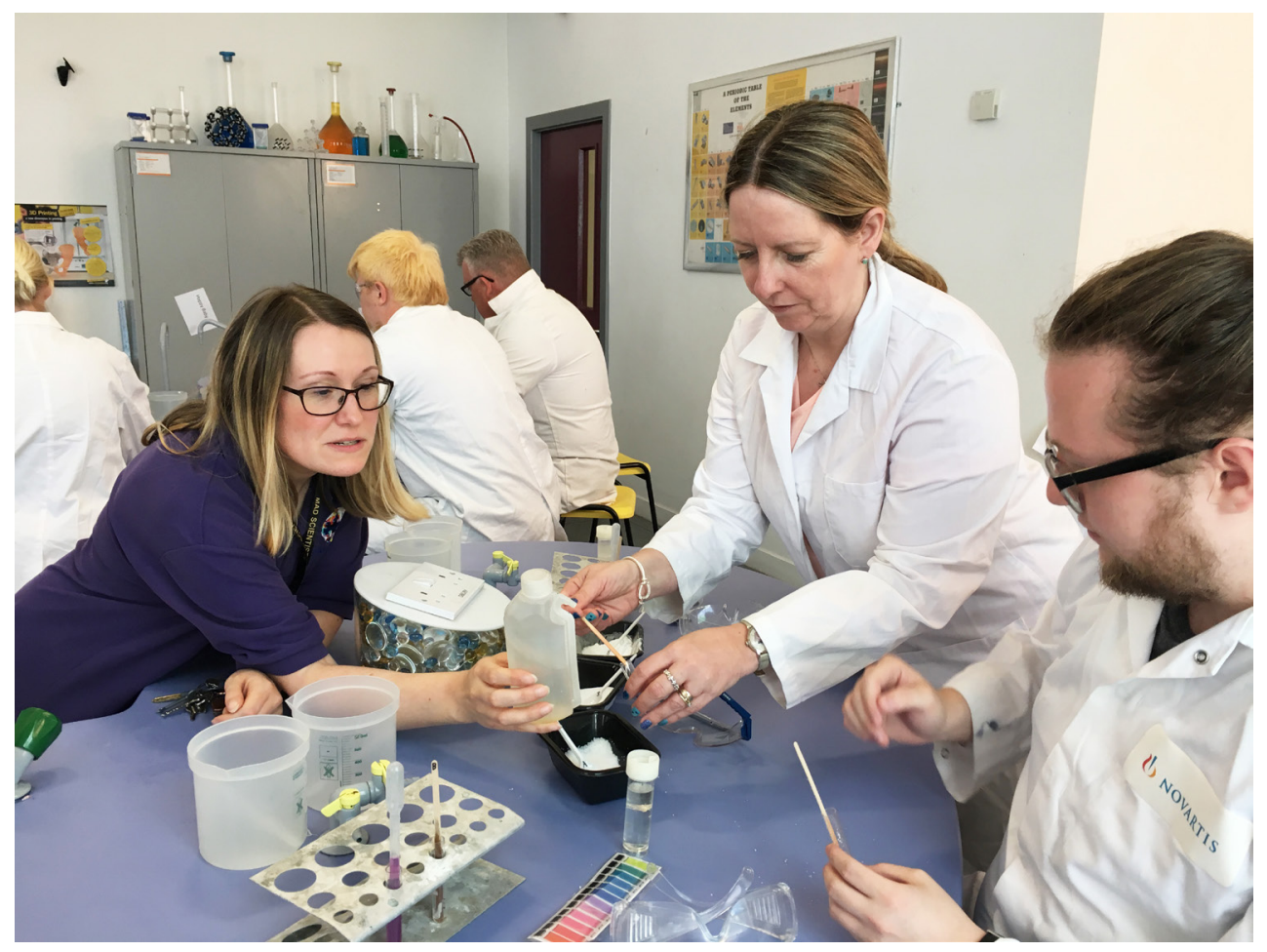

Image 3. MindLab project workshop in the lab, 2019

\section{During and After: Challenges and Opportunities}

As an independent charity, Catalyst receives no government financial support. It relies on income generated through school visits, sleepovers, admissions, the shop/café, and grant funding. Like many museums at the time of writing, Catalyst has furloughed all but one of its staff, leaving the challenge of continuing projects with a reduced team, resulting in increased 
anxiety among staff who feel they are 'doing nothing' to help communities in need. Social distancing has also affected the ways in which this project can be delivered. Without knowing exactly what measures will be in place in the future, devising solutions to deliver activities safely is challenging, particularly when support groups rely on a level of intimacy.

In late April 2020, with new health and safety plans and protocols in place, we began reshaping MindLab in order to respond to the community's changing needs and priorities. This was done in collaboration with Mind Halton, which during the lockdown continued providing support through a telephone 'listening ear' service, as well as consulting Catalyst's network of schools and education professionals. What emerged were strong concerns regarding young people and their mental health during and post lockdown. The disruption to the daily lives of young people caused by school closures is unprecedented, with 83 per cent of the young people surveyed by YoungMinds ${ }^{6}$ reporting that the pandemic had worsened their mental health conditions.

In June 2020 Catalyst gained a grant from the INEOS Community Fund to continue work with local adults. We are also expanding this project to support young people by partnering with charity YouthFed, which offers wellbeing and mentoring services locally. Although we remain closed to the general public, in July 2020 we started welcoming small pre-booked MindLab groups back into Catalyst. In September 2020 we delivered a digital science-inspired offer with YouthFed to complement their existing online wellbeing support.

To conclude, while a growing body of evidence indicates that cultural participation, namely participation with museums and art, has the power to enhance health and wellbeing (Konlaan et al. 2000; Chatterjee and Noble 2013; Napier et al. 2014), there has been less attention to the role of science engagement. This project suggests that science does have potential to support wellbeing; we hope our work can contribute to research in this area.

Maintaining strong local partnerships is the foundation for supporting our community. This has enabled us to stay connected and relevant to their needs during and after lockdown. We therefore hope this article contributes to both practical and scholarly discourses as to how small, local museums can deliver social impact.

Received: 12 June 2020

Finally accepted: 21 August 2020

\section{Notes}

1 Office of National Statistics, 'Coronavirus and the Social Impacts on Great Britain: 16 April 2020', 2020. https://www.ons.gov.uk/peoplepopulationandcommunity/healthandsocialcare/ healthandwellbeing/bulletins/coronavirusandthesocialimpactsongreatbritain/16april2020, accessed 13 May 2020.

2 Made possible through the Inspiring Science Fund, funded by BEIS and Wellcome Trust.

3 Ministry of Housing, Communities and Local Government, 'English Indices of Deprivation 2019: Research Report', 2019. https://www.gov.uk/government/statistics/english-indicesof-deprivation-2019, accessed 13 May 2020.

4 World Health Organization, 'Investing in Mental Health: Evidence for Action', 2013. https:// apps.who.int/iris/handle/10665/87232, accessed 13 May 2020.

5 Mind, 'Five Ways to Wellbeing', 2020. https://www.mind.org.uk/workplace/mental-healthat-work/taking-care-of-yourself/five-ways-to-wellbeingl, accessed 28 July 2020.

6 YoungMinds, 'Coronavirus: Impact on Young People with Mental Health Needs Report', 2020. https://youngminds.org.uk/about-us/reports/coronavirus-impact-on-young-peoplewith-mental-health-needs, accessed 13 May 2020; Office of National Statistics, 'Coronavirus and the Social Impacts on Great Britain'. 


\section{References}

Chatterjee, H.J. and Noble, G. (2013) Museums, Health and Well-being, Farnham, Surrey: Ashgate Publications.

Konlaan, B.B., Bygren, L.O. and Johansson, S.E. (2000) 'Visiting the Cinema, Concerts, Museums or Art Exhibitions as Determinant of Survival: A Swedish Fourteen-year Cohort Follow-up', Scandinavian Journal of Public Health, 28 174-78. doi:10.1177/14 034948000280030501

Napier, A.D. et al. (2014) 'Culture and Health', The Lancet, 384 1607-39. doi:10.1016/ S0140-6736(14) 61603-2

\section{Authors}

\section{Jade French}

ORCID iD: @ https://orcid.org/0000-0003-0067-6169

URL: https://ahc.leeds.ac.uk/fine-art/staff/271/dr-jade-french

University of Leeds

United Kingdom

j.french2@leeds.ac.uk

Visiting Research Fellow in the School of Fine Art, History of Art and Cultural Studies and Community Engagement Coordinator at Catalyst Science Discovery Centre.

\section{Nic Lunt}

URL: http://www.mindhalton.org.uk/

Mind Halton

United Kingdom

nic@mindhalton.org.uk

Chief Executive Officer at Mind Halton.

\section{Martin Pearson}

URL: https://catalyst.org.uk/

Catalyst Science Discovery Centre

United Kingdom

martinceo@catalyst.org.uk

Chief Executive Officer at Catalyst Science Discovery Centre. 